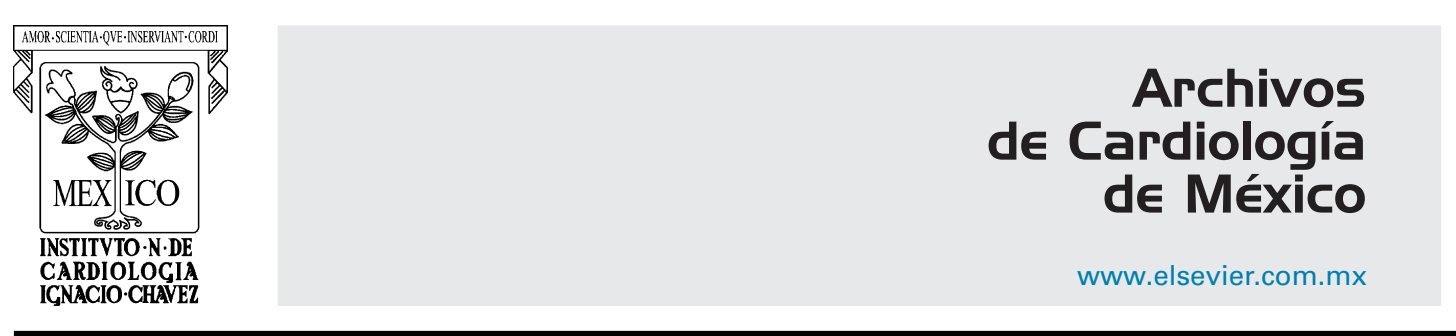

INVESTIGACIÓN CLÍNICA

\title{
Estratificación del dolor torácico con el score HEART modificado y su relación con eventos adversos cardiovasculares a corto plazo
}

\author{
Manuel Chacón-Diaz*, Jorge Salinas y Rafael Doig
}

Servicio de cardiología, Clínica Delgado, Lima, Perú

Recibido el 19 de abril de 2017; aceptado el 19 de junio de 2017

\author{
PALABRAS CLAVE \\ Dolor torácico; \\ Score HEART \\ modificado; \\ Eventos adversos; \\ Perú
}

\begin{abstract}
Resumen
Objetivo: El dolor torácico es un importante motivo de atención en salas de urgencia a nivel mundial. Se evaluó la relación entre el score HEART modificado y la presencia de eventos cardiacos mayores a los 30 días.

Métodos: Estudio retrospectivo, observacional en un solo centro hospitalario. En pacientes mayores de 18 años atendidos en urgencias por dolor torácico, en los cuales se aplicó el score HEART modificado al ingreso y se relacionó con la presencia de eventos cardiacos mayores (infarto de miocardio, muerte, re hospitalización por causa cardiaca y revascularización coronaria percutánea o quirúrgica) a los 30 días de seguimiento.

Resultados: De 158 pacientes analizados, 17 eventos adversos (10.8\%) se encontraron al mes de seguimiento. El score HEART modificado pudo predecir eventos adversos en el 4; 21,4 y $100 \%$ de pacientes con scores $0-3,4-6$ y $7-10$ respectivamente $(p=0.0001)$. Un score HEART modificado mayor o igual a 4 se relacionó con más eventos adversos (OR: 4.52; IC: 2.76-7.39) con una sensibilidad del $70 \%$ y una especificidad del $84 \%$.

Conclusiones: La aplicación del score HEART modificado estratifica a los pacientes con dolor torácico en urgencias de manera adecuada en bajo, moderado y alto riesgo de complicaciones cardiovasculares, lo cual permite que las unidades de urgencia mejoren sus protocolos de triaje y diagnóstico de los síndromes coronarios agudos.

(c) 2017 Instituto Nacional de Cardiología Ignacio Chávez. Publicado por Masson Doyma México S.A. Este es un artículo Open Access bajo la licencia CC BY-NC-ND (http://creativecommons. org/licenses/by-nc-nd/4.0/).
\end{abstract}

\footnotetext{
* Autor para correspondencia. Calle Coronel Inclan 425, departamento 401, Miraflores, Lima 18, Perú. Teléfono: +051 14555456 ; móvil: 0511989433241.

Correo electrónico: manuelchacon03@yahoo.es (M. Chacón-Diaz).
} 


\section{KEYWORDS}

Chest pain; Modified HEART

Score;

Adverse events;

Peru
Stratification of thoracic pain with modified HEART score and its relationship to short term cardiovascular events

\begin{abstract}
Objective: Chest pain is a major reason for emergency room care worldwide. The relationship between the Modified Heart Score and the presence of major cardiac events at 30 days after emergency admission was evaluated.

Methods: Retrospective, observational study in a single centre on patients older than 18 years, who were treated for chest pain. The Modified HEART Score was applied at admission and related to the presence of major cardiac events (myocardial infarction, death, hospital re-admission due to cardiac causes, and percutaneous or surgical coronary revascularisation) at 30 days of follow-up.

Results: Of 158 patients analysed, $17(10.8 \%)$ adverse events were found at follow-up. The modified HEART score could predict adverse events in $4 \% ; 21.4 \%$, and $100 \%$ of patients with scores $0-3 ; 4-6$, and 7-10, respectively $(P=.0001)$. A modified HEART score greater than or equal to 4 was associated with more adverse events (OR: 4.52 ; $95 \% \mathrm{Cl} 2.76-7.39$ ) with a sensitivity of $70 \%$ and specificity of $84 \%$.

Conclusions: The application of the modified HEART score is useful for stratifying patients with chest pain into low, moderate, and high risk of cardiovascular complications, which should help the emergency units to improve their protocols for triage and diagnosis of acute coronary syndromes.

(C) 2017 Instituto Nacional de Cardiología Ignacio Chávez. Published by Masson Doyma México S.A. This is an open access article under the CC BY-NC-ND license (http://creativecommons. org/licenses/by-nc-nd/4.0/).
\end{abstract}

\section{Introducción}

El dolor torácico es una de las primeras causas de atención en salas de urgencia a nivel mundial. Uno de los objetivos de su manejo es distinguir los síndromes coronarios agudos de otras causas de dolor torácico.

Las guías para el diagnóstico y tratamiento del síndrome coronario agudo sin elevación del $\mathrm{ST}^{1}$ han definido los criterios para poder hacer un diagnóstico oportuno de esta entidad, sobre todo con la presencia de cambios electrocardiográficos y elevación de enzimas cardiacas. En el caso de diagnóstico del síndrome coronario agudo con elevación del ST el diagnóstico es más sencillo por las características del electrocardiograma ${ }^{2}$.

Sin embargo, muchas veces no es posible excluir un síndrome coronario agudo sin elevación del ST con tan solo la ausencia de cambios electrocardiográficos y/o de enzimas cardiacas, llevando muchas veces a hospitalizaciones innecesarias, y peor aún, a dar de alta a pacientes que en realidad se encontraban con un evento coronario en curso. Por este motivo Six et al. ${ }^{3}$ llevaron a cabo un estudio clínico el año 2008 en Holanda para estratificar a pacientes con dolor torácico en salas de urgencia (excluyendo pacientes con ST elevado). De este estudio se deriva el score HEART; con la utilización de 5 parámetros de fácil acceso en urgencia desarrollaron el score que demostró utilidad en predecir infarto de miocardio, revascularización coronaria y muerte a más de un año de seguimiento en pacientes con score mayor a 7.

Posteriormente se realizó la validación multinacional (región Asia-Pacífico) de este score con 2,906 pacientes, agrupándolos en 3 grupos: bajo riesgo (score HEART de 0 -
3), riesgo intermedio (score de 4-6) y alto riesgo (score de 7 a 10). Se encontró mayor incidencia de infarto de miocardio, revascularización coronaria o muerte a 30 días (el 1.7\% en pacientes de bajo riesgo versus el $43 \%$ en el grupo de alto riesgo) y se demostró que era mejor que el score TIMI en predecir síndromes coronarios agudos en pacientes con dolor torácico en la urgencia ${ }^{4}$. Además este score TIMI se usa para estratificar a pacientes con síndrome coronario agudo con ST no elevado ya confirmado. Varios estudios ${ }^{5}$ han confirmado a nivel mundial la validez de este score, inclusive con algunas variaciones incluyendo la troponina I ultrasensible ${ }^{6}$; este último score es el que se va a valorar en el presente estudio (tabla 1).

\section{Métodos}

Estudio retrospectivo, observacional en un solo centro hospitalario. El objetivo general fue relacionar el score HEART modificado con la presencia de eventos adversos (infarto de miocardio, muerte, rehospitalización por dolor torácico de causa cardiaca y revascularización coronaria percutánea o quirúrgica) a 30 días de seguimiento tras el ingreso a urgencias por dolor torácico.

Se incluyen pacientes mayores de 18 años de edad con dolor torácico ingresados por urgencias de la clínica Delgado durante enero a diciembre del año 2016. Se excluyen pacientes con diagnóstico de síndrome coronario agudo con elevación del segmento ST, embolia pulmonar, síndrome aórtico agudo, trauma torácico, neumonía y gestantes. La revisión de los electrocardiogramas fue hecha por el investigador principal; la caracterización del dolor se realizó 
Tabla 1 Score HEART modificado

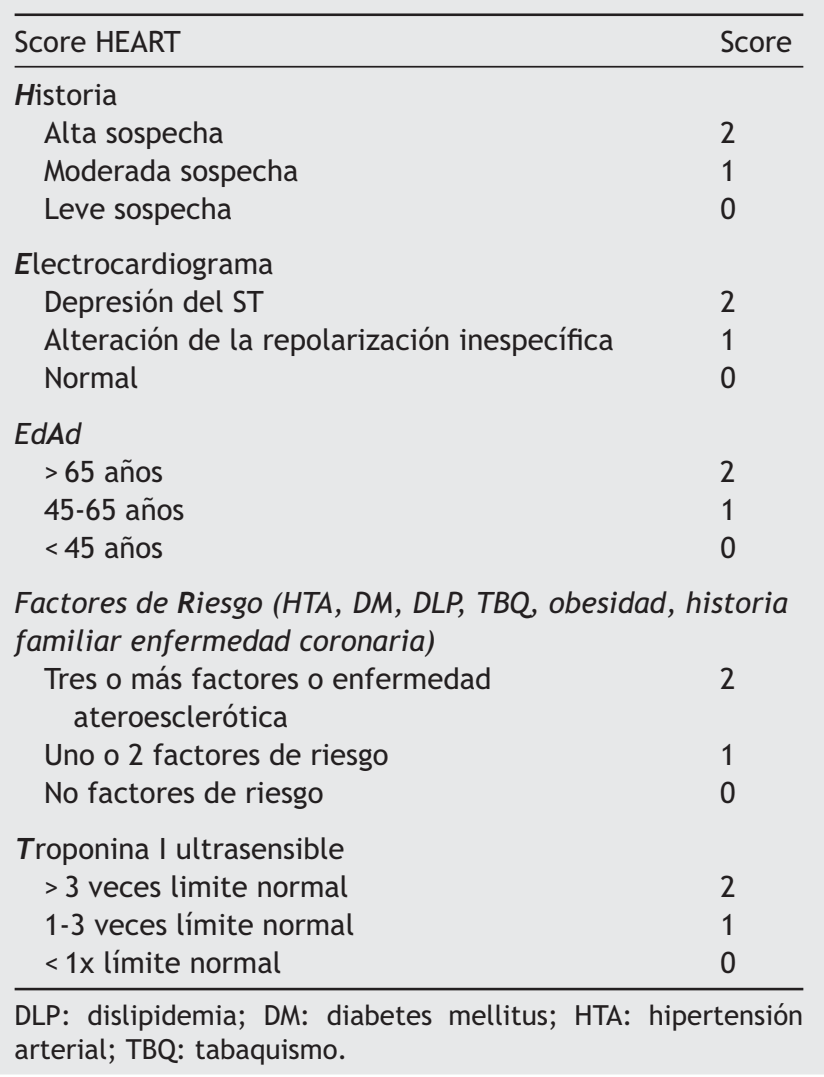

tomando en cuenta lo descrito en la historia clínica por el médico de urgencia.

El procesamiento de datos se llevó a cabo tomando en cuenta la frecuencia de las variables, porcentajes, promedios, medias y desviación estándar para datos con distribución normal, y medianas y rango intercuartílico para variables sin distribución normal. Para la evaluación de asociación entre variables cualitativas se utilizó la prueba de Chi-cuadrado, para variables cuantitativas la prueba $T$ de Student. Se consideró significativo un resultado de $p<0.05$. Todos los análisis fueron realizados utilizando el paquete estadístico SPSS versión 22.

\section{Resultados}

Durante enero a diciembre 2016 se atendieron un total de 283 pacientes con síntomas de dolor torácico; aplicando los criterios de exclusión la población final de estudio fue de 158 pacientes (fig. 1). Un 62.7\% fueron varones, la edad media fue de $49.4 \pm 13.4$ años (mínima 26 y máxima 89 años). Un $42 \%$ (67 pacientes) ingresaron con cuadro de dolor precordial catalogado como opresivo retroesternal con o sin irradiación a otras áreas. Otros tipos de dolor fueron clasificados como punzada (48 pacientes), quemazón ( 5 pacientes) o urente (38 pacientes).

El electrocardiograma al ingreso fue normal en el $84.8 \%$ de casos, el $14.5 \%$ con trastorno de repolarización inespecífico y un solo caso $(0.6 \%)$ con infradesnivel del segmento ST.

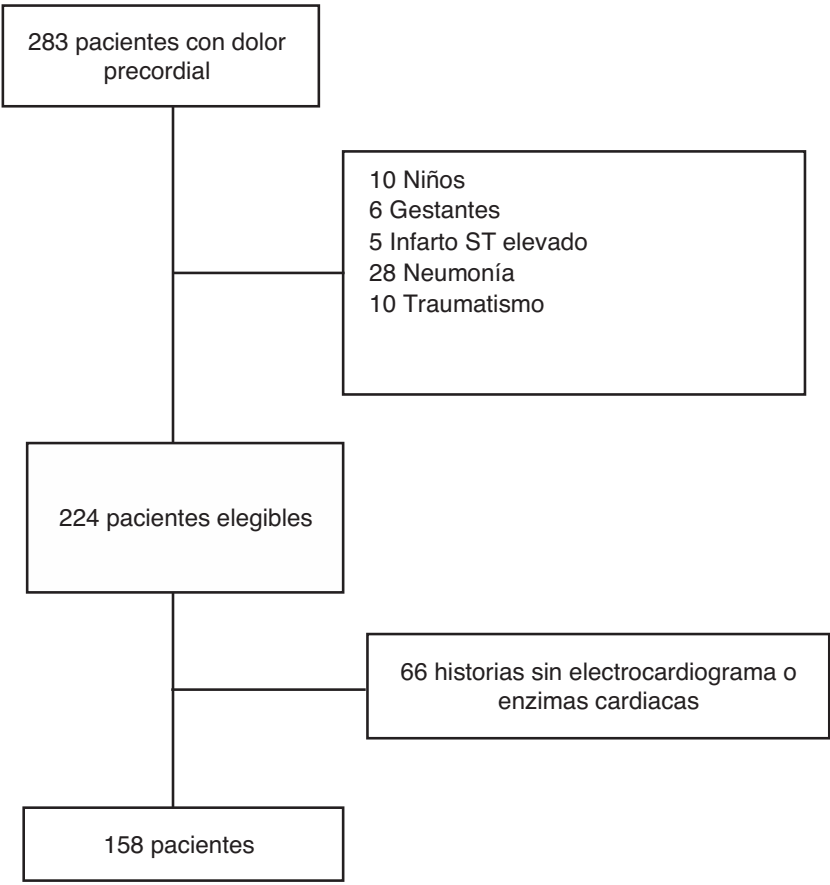

Figura 1 Cuadro de flujo de los participantes del estudio.

La troponina T ultrasensible fue tomada al momento de ingreso del paciente a urgencias y se encontró en valores normales en el $\mathbf{9 5 . 5 \%}$ de los casos. El lapso entre el inicio de síntomas y la toma de muestra fue de $5 \mathrm{~h}$ (rango intercuartílico: 2-13.3). Solo se tomó una muestra de troponina T ultrasensible en los casos con más de $4 \mathrm{~h}$ de evolución, en el resto de los casos se tomo una nueva muestra al cumplir las $4 \mathrm{~h}$ de inicio de síntomas y se consideró el valor más alto para el análisis.

En general 137 pacientes (86.7\%) fueron dados de alta sin mayor estratificación, a 12 pacientes (7.6\%) se les estratificó no invasivamente y 9 pacientes $(5.7 \%)$ recibieron manejo y estratificación invasiva (coronariografía), 4 de estos con score HEART de alto riesgo y 5 con score intermedio, con el $85 \%$ ( 6 pacientes) debido a un resultado de troponina $T$ elevada.

Al aplicar el score HEART modificado, se encontró que hubo $124(78.5 \%), 28(17.7 \%)$ y $6(3.8 \%)$ pacientes con score 0-3, 4-6 y 7-10 respectivamente.

Las características demográficas de los pacientes según los grupos de riesgo del score HEART modificado están presentadas en la tabla 2.

Diecisiete pacientes $(10.8 \%)$ presentaron eventos adversos a los 30 días de seguimiento. La edad media en el grupo con eventos adversos fue de 60.3 años versus 48.1 años en el grupo sin eventos adversos $(p=0.006)$; no se encontraron diferencias por sexo. La presencia de hipertensión arterial, dislipidemia, infarto de miocardio previo y enfermedad coronaria crónica aumentaron el riesgo de eventos adversos; así, el OR fue de 3.04 (IC: 1.09-8.47) $(p=0.027)$ para la hipertensión arterial, OR de 4.7 (IC: 1.6-13.4) $(p=0.004)$ para dislipidemia, OR de 14 (IC: 3.37-60.3) $(\mathrm{p}=0.001)$ para el antecedente de infarto de miocardio previo y OR de 12.2 (IC: $3.38-44.4)(p=0000)$ para el antecedente de enferme- 
Tabla 2 Características de la población según el score HEART modificado

\begin{tabular}{|c|c|c|c|c|}
\hline Score HEART modificado & $\begin{array}{l}\text { Bajo (0-3) } \\
\text { n (\%) }\end{array}$ & $\begin{array}{l}\text { Moderado } \\
(4-6) \\
n(\%)\end{array}$ & $\begin{array}{l}\text { Alto (7-10) } \\
\text { n (\%) }\end{array}$ & Valor $p$ \\
\hline Varones & $79(63.7)$ & $14(50)$ & $6(100)$ & 0.062 \\
\hline Edad (años) & $45 \pm 10.4$ & $63 \pm 13.2$ & $67 \pm 8.7$ & 0.001 \\
\hline Hipertensión & $26(21.4)$ & $18(64.2)$ & $3(50)$ & 0.001 \\
\hline Diabetes & $5(4.2)$ & $8(28.1)$ & $1(16)$ & 0.001 \\
\hline Dislipidemia & $15(12)$ & $17(60)$ & $2(66.7)$ & 0.000 \\
\hline Infarto previo & $7(1.6)$ & $2(7.1)$ & $5(83.3)$ & 0.000 \\
\hline Tabaquismo & $9(7.3)$ & $5(17.9)$ & $1(16.7)$ & 0.230 \\
\hline Obesidad & $7(5.6)$ & $4(14.3)$ & - & 0.219 \\
\hline Antecedentes familiar & $2(1.6)$ & - & - & 0.614 \\
\hline ECOC & $3(2.4)$ & $4(14.3)$ & $5(83.3)$ & 0.000 \\
\hline Dolor opresivo & $39(31.5)$ & $22(78.6)$ & $6(100)$ & 0.000 \\
\hline Nitratos & - & $11(39.3)$ & $4(66.7)$ & 0.001 \\
\hline Antiagregantes & $8(6.5)$ & $20(71.4)$ & $4(66.7)$ & 0.001 \\
\hline Anticoagulación & - & $5(17.9)$ & $4(66.7)$ & 0.001 \\
\hline Analgésicos & $88(71)$ & $6(21.4)$ & - & 0.000 \\
\hline Estratificación Invasiva & - & $5(17.9)$ & $4(66.7)$ & 0.000 \\
\hline Estratificación no invasiva & $7(5.6)$ & $5(17.9)$ & - & 0.068 \\
\hline Alta sin estratificación & $117(94.4)$ & $18(64.3)$ & $2(33.3)$ & 0.000 \\
\hline
\end{tabular}

ECOC: enfermedad coronaria obstructiva crónica como antecedente en la anamnesis.

Tabla 3 Eventos adversos a 30 días, según score de HEART modificado

\begin{tabular}{|c|c|c|c|c|}
\hline Score HEART modificado & $\begin{array}{l}\text { Bajo (0-3) } \\
\text { n (\%) }\end{array}$ & $\begin{array}{l}\text { Moderado } \\
(4-6) \\
\mathrm{n}(\%)\end{array}$ & $\begin{array}{l}\text { Alto }(7-10) \\
n(\%)\end{array}$ & Valor $\mathrm{p}$ \\
\hline Mortalidad & - & - & - & - \\
\hline Infarto de miocardio & - & $1(3.6)$ & $1(16.7)$ & 0.025 \\
\hline Revascularización & - & $3(10.7)$ & $6(100)$ & 0.000 \\
\hline $\mathrm{PCl}$ & - & $2(7.1)$ & $6(100)$ & 0.000 \\
\hline CABG & - & $1(3.6)$ & - & 0.175 \\
\hline Rehospitalización & $5(4)$ & $4(14.3)$ & $3(50)$ & 0.003 \\
\hline Compuesto & $5(4)$ & $6(21.4)$ & $6(100)$ & 0.000 \\
\hline
\end{tabular}

CABG: cirugía de revascularización coronaria; PCI: angioplastia coronaria percutánea.

dad coronaria obstructiva crónica. El resto de antecedentes no tuvieron significación estadística.

El dolor torácico muy sugestivo (según la clasificación del score HEART modificado) se presentó en el $58.8 \%$ de los pacientes con eventos adversos versus el $18.4 \%$ sin eventos adversos $(p=0.000)$.

En el grupo de pacientes con eventos adversos un 5.9\% tuvieron infradesnivel del segmento ST en el electrocardiograma, un $47.1 \%$ trastorno de repolarización ventricular y un $47.1 \%$ fue normal. A diferencia de los que no tuvieron eventos adversos entre los que el $89.4 \%$ de casos tuvieron electrocardiogramas normales y el $10.6 \%$ con trastorno de repolarización ventricular $(p=0.000)$. El $64.7 \%$ de los pacientes con eventos adversos tuvieron valores de troponina dentro de rango normal.

Los eventos adversos según el score HEART modificado se presentan en la tabla 3 y en la figura 2). Se encontró mayor porcentaje de eventos conforme aumentó el score (fig. 3).
No encontramos casos de mortalidad a 30 días. Se presentaron 2 casos de infarto de miocardio tras ser dados de alta, uno de ellos de riesgo moderado tras ser dado de alta sin mayor estratificación y el otro en un paciente con riesgo alto manejado invasivamente que regresó por trombosis de stent. El $41.7 \%$ de los pacientes que reingresaron por dolor torácico a los 30 días tuvieron un score bajo al inicio.

La revascularización coronaria percutánea se practicó en 8 pacientes de los cuales 6 tuvieron score HEART de alto riesgo (4 de ellos con diagnóstico de infarto sin elevación del segmento ST en la hospitalización índice y 2 tras su reingreso por síndrome coronario agudo dentro del mes del evento inicial).

La revascularización en el grupo con score HEART de riesgo intermedio se dio en 3 de los 5 pacientes que fueron a estrategia invasiva: 2 lo hicieron vía percutánea por ser troponino positivos y en un caso vía quirúrgica; en los 2 pacientes restantes no se encontró lesiones coronarias susceptibles de revascularización. 


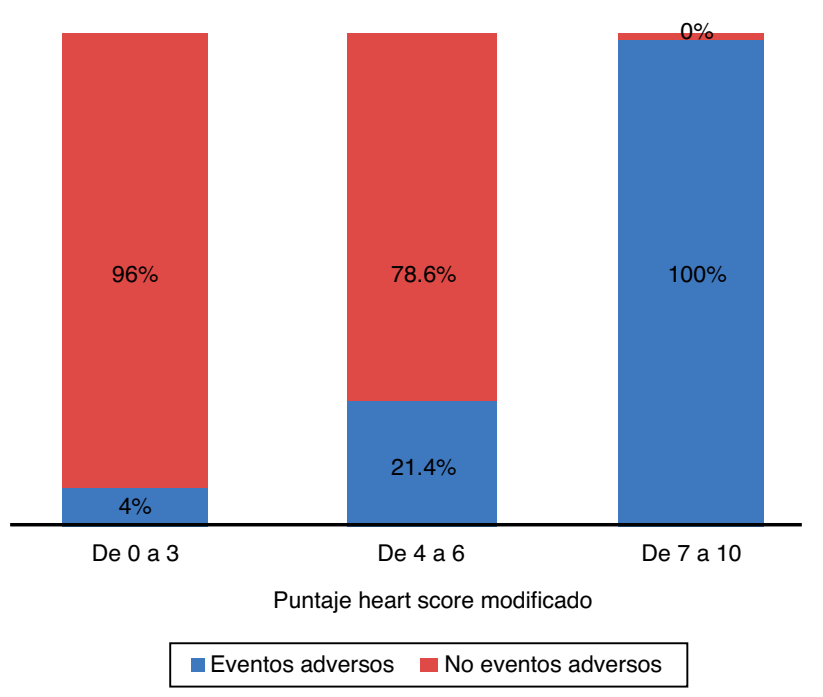

Figura 2 Eventos adversos a 30 días según puntaje del score HEART modificado.

HEART: historia, electrocardiograma, Edad, factores de riesgo, troponina.

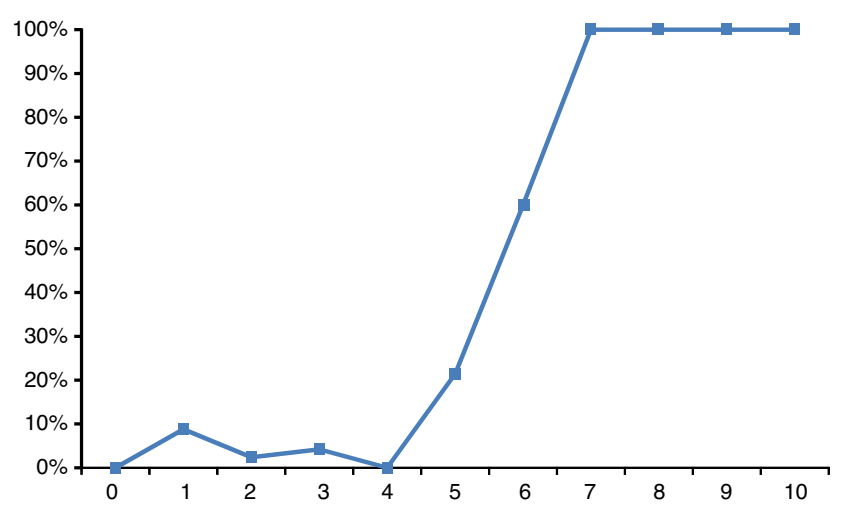

Figura 3 Aumento de eventos adversos con el aumento del puntaje del score HEART modificado. $(p=0.001)$.

HEART: historia, electrocardiograma, Edad, factores de riesgo, troponina.

En cuanto a la rehospitalización por dolor torácico, 3 pacientes con score HEART de alto riesgo fueron rehospitalizados: uno por trombosis de stent y los otros 2 por angina inestable.

En el caso de pacientes con score intermedio, 4 pacientes se rehospitalizaron: uno por angina sin lesiones coronarias y los otros 3 por cuadros de dolor torácico catalogados como angina inestable. En pacientes con score HEART bajo, 5 se rehospitalizaron: 3 tras exámenes de isquemia negativos (ergometría) y 2 tras ser dados de alta sin más estudios; el motivo de reingreso fue dolor torácico opresivo por pericarditis ( 2 casos) y el resto por dolor precordial no cardiaco.

Llama la atención la baja proporción de pacientes con riesgo intermedio a los que se realizó estratificación no invasiva (ergometría a 3 pacientes, SPECT a un paciente y tomografía coronaria a un paciente) que llegó a solo un $17.9 \%$. En los pacientes con score de bajo riesgo se hicieron 7 ergometrías como estratificación no invasiva, 3 de ellos reingresaron por dolor torácico dentro del primer mes y los otros 4 no tuvieron eventos al seguimiento. La decisión de coronariografía según los datos de los test no invasivos fue tomada según las guías actuales. Un $33 \%$ de pacientes con score HEART alto fueron dados de alta sin mayor estratificación.

Al clasificar a los pacientes en aquellos con un score HEART modificado mayor o igual a 4 y otro grupo con score menor a 4, se encontró un 35.3 vs. $4 \%$ con eventos adversos respectivamente; OR 4.52 (IC: 2.76-7.39) $p=0.000$, obteniendo una sensibilidad del $70 \%$ y una especificidad del $84 \%$ para predecir eventos adversos a 30 días con un score mayor o igual a 4.

En cambio, al comparar los eventos adversos de pacientes con score mayor o igual a 7 , se encontró que el $100 \%$ de estos tuvieron eventos versus el $7.2 \%$ con score menor a 7 ; con una especificidad del $100 \%$ pero una sensibilidad de tan solo el $35 \%$.

\section{Discusión}

El manejo del dolor torácico en urgencias supone realizar los procedimientos de descarte de enfermedad cardiaca o pulmonar de riesgo, con miras a descartar síndromes coronarios agudos u otras enfermedades que pongan en riesgo la vida de la persona.

El score HEART fue desarrollado con miras a utilizar un score fácil de recordar y que pueda ser aplicado por médicos con poca o mucha experiencia ${ }^{7}$, con el uso de 5 parámetros y cada uno de ellos dividido en 3 posibles resultados con puntajes de 0 a 2 . Este score es un potente detector de pacientes de bajo riesgo ${ }^{4}$; así en nuestro estudio el valor predictivo negativo de un score mayor 4 es de $95 \%$.

Tomando en cuenta los niveles de riesgo según el score de 0-3 riesgo bajo, 4-6 riesgo intermedio y $7-10$ riesgo alto de eventos cardiacos mayores, encontramos que el $100 \%$ de los pacientes con score de alto riesgo tuvieron eventos adversos y solo $4 \%$ de los de bajo riesgo. Backus et al. ${ }^{7}$ encontraron la presencia de eventos adversos con un score de bajo riesgo del $0.99 \%$, del $11.6 \%$ con los de riesgo intermedio y del $65.2 \%$ en los de alto riesgo. Es importante mencionar que en el presente estudio el score fue más significativo en predecir infarto de miocardio, revascularización y rehospitalización por dolor torácico de origen cardiaco (síndrome coronario agudo), no encontrando casos de mortalidad.

Se utilizó la dosificación de troponina ultrasensible debido a su mayor capacidad diagnóstica, siendo su límite menor de detección de $5 \mathrm{ng} / \mathrm{L}$ versus $10 \mathrm{ng} / \mathrm{L}$ de la troponina convencional ${ }^{8}$. Para Willems et al. ${ }^{8}$ un valor negativo de troponina T ultrasensible asociada a un score HEART bajo permitiría dar el alta con seguridad a los pacientes con 4-6 h de inicio de dolor. En el presente estudio se encontró que el 92.7\% de los pacientes con troponina normal no presentaron eventos adversos.

La importancia del estudio radica en que demuestra la utilidad de poner en práctica el score HEART en las salas de urgencias, que sea este aplicado por el médico de urgencias o el cardiólogo de turno, con miras a estratificar de mejor manera a los pacientes con dolor torácico, evitando la sobrecarga de pacientes hospitalizados con bajo riesgo y sobre todo el alta de pacientes de alto riesgo. 
Las limitaciones de este estudio son: su carácter retrospectivo, el tamaño de muestra reducido, el hecho de que los datos hayan sido tomados por diferentes médicos lo cual puede sesgar la puntuación final del score, la baja proporción de pacientes con eventos adversos a 30 días y que fue realizado en un solo centro asistencial.

\section{Conclusión}

La aplicación del score HEART modificado estratifica a los pacientes con dolor torácico en urgencias de manera adecuada en bajo, moderado y alto riesgo de complicaciones cardiovasculares, lo cual permite que las unidades de urgencias mejoren sus protocolos de triaje y diagnóstico de los síndromes coronarios agudos.

\section{Responsabilidades éticas}

Protección de personas y animales. Los autores declaran que para esta investigación no se han realizado experimentos en seres humanos ni en animales.

Confidencialidad de los datos. Los autores declaran que han seguido los protocolos de su centro de trabajo sobre la publicación de datos de pacientes.

Derecho a la privacidad y consentimiento informado. Los autores declaran que en este artículo no aparecen datos de pacientes.

\section{Financiación}

Ninguna.

\section{Conflicto de intereses}

Los autores declaran no tener ningún conflicto de intereses.

\section{Bibliografía}

1. Roofi M, Patrono C, Collet J, et al. 2015 ESC guidelines for the management of acute coronary syndromes in patients presenting without persistent ST-segment elevation: Task Force for the Management of Acute Coronary Syndromes in Patients Presenting without Persistent ST-Segment Elevation of the European Society of Cardiology (ESC). Eur Heart J. 2016;37: 67-315.

2. Steg G, James S, Atar D, et al. ESC Guidelines for the management of acute myocardial infarction in patients presenting with ST-segment elevation: The Task Force on the management of ST-segment elevation acute myocardial infarction of the European Society of Cardiology (ESC). Eur Heart J. 2012;33: 2569-619.

3. Six AJ, Backus B, Kelder J. Chest pain in the emergency room: Value of the HEART score. Neth Heart J. 2008;16: 191-6.

4. Six AJ, Cullen L, Backus B, et al. The HEART Score for the Assessment of Patients With Chest Pain in the Emergency Department. A Multinational Validation Study. Crit Pathw Cardiol. 2013;12:121-6.

5. Poldervaart J, Reitsma J, Koffijberg $\mathrm{H}$, et al. The impact of the HEART risk score in the early assessment of patients with acute chest pain: Design of a stepped wedge, cluster randomised trial. BMC Cardiovasc Disord. 2013;13:77-84.

6. Chun-Peng MA, Xiao WANG, Qing-Shung WANG, et al. A modified HEART risk score in chest pain patients with suspected non-STsegment elevation acute coronary syndrome. J Geriatr Cardiol. 2016;13:64-9.

7. Backus B, Six A, Kelder J, et al. Chest pain in the emergency room: A multicenter validation of the HEART Score. Crit Pathw Cardiol. 2010;9:164-9.

8. Willems $M$, van de Wijngaart $D$, Bergman $H$, et al. Addition of heart score to high-sensitivity troponin $\mathrm{T}$ versus conventional troponin $T$ in risk stratification of patients with chest pain at the coronary emergency rooms. Netherland Heart Journal. 2014;22:552-6. 